\title{
A Dynamic and Highly Configurable Crypto-Processor for brief communication interval
}

\author{
${ }^{* 1}$ Riya Bagul, Atharva Karaguppi ${ }^{2}$, Vishwas Karale ${ }^{3}$, Mudit Singal ${ }^{4}$, Dr. Vaishali \\ Ingale ${ }^{5}$ \\ ${ }^{1}$ Electronics and Telecommunication, College of Engineering, Pune \\ ${ }^{2}$ Electronics and Telecommunication, College of Engineering, Pune \\ ${ }^{3}$ Electronics and Telecommunication, College of Engineering, Pune \\ ${ }^{4}$ Electronics and Telecommunication, College of Engineering, Pune.
}

D.O.I - 10.51201/JUSST/21/05162

\begin{abstract}
In modern computing systems data security is of paramount importance. The data transfer must be made secure because it can be significantly sensitive for any organization involved. This paper expounds a SOC architecture to facilitate end to end secure data exchange for applications involving short communication intervals. This SOC has been designed to behave as a co-processor which along with a standard general-purpose processor would serve as a cryptosystem. The SOC employs two famous algorithms - RSA and AES for cryptography. In contrast to usual single key cryptographic systems, this paper tries to elaborate an innovative methodology involving dynamic security measures that makes the system distributed rather than making it central to a specific algorithm and hence a particular key. The methodology involves generating and using an AES key for data encryption and RSA key for secure transfer of the AES key between the point of transmission and reception.
\end{abstract}

Keywords: Cryptography, encryption, FPGA (Field Programmable Gate Array), RSA (Rivest Shamir Adleman), AES (Advanced Encryption Standard), SOC (System on Chip), Artix-7

\section{INTRODUCTION}

This paper elaborates an SOC architecture that has been designed and implemented on an Artix7 FPGA (XC7ATCSG324-2L) to act as cryptosystem. This system has been designed to target applications where the communication time is small, for instance satellite applications. This is a typical example where the time of communication is limited by the duration in which the satellite is in the line of sight of the ground station. Such an application poses the need for a fast and secure system. The SOC, which will act as a co-processor module, shall communicate with a main processor using an UART link which would be responsible for data transfer for carrying out encryption and decryption operations. The main processor would be responsible for processing and fetching the necessary data before encryption in addition to obtaining encrypted requests from the receiver to be fed to the SOC for decryption and further processing.

A strategy similar to the Diffie Hellman exchange has been developed in order to establish an effective and secure communication link between the transmitter and the receiver. Figure 1 
illustrates the procedure involved for establishing a communication link between the receiver and transmitter.

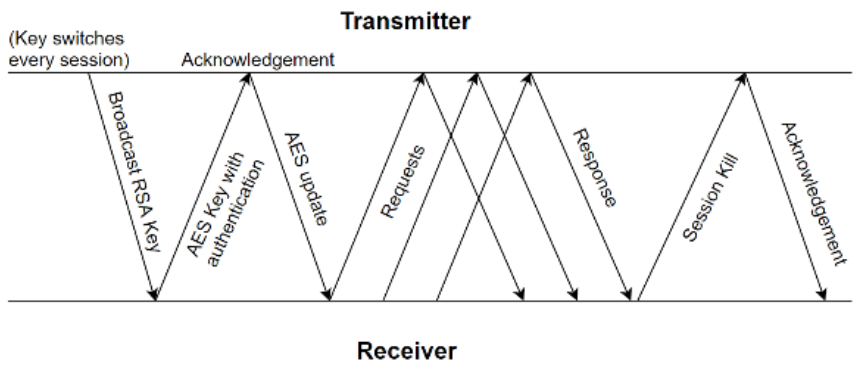

Figure 1

To begin with, the transmitter generates and broadcasts an RSA public key which would be picked up by the receiver. The receiver generates an AES key which is then transmitted by encrypting it using the RSA public key. After receiving an acknowledgement from the receiver, the response is decrypted using the corresponding RSA private key at the transmitter end to obtain the AES key. Now a secure link has been established. This AES key acts as the cryptographic link for all the future data transactions. The requests from the receiver, encrypted with AES are decrypted and processed at the transmitter end. The requested data is encrypted with AES and then transmitted. This request and data exchange between the receiver and the transmitter continues until the receiver sends a session kill command to the transmitter which is a followed by an acknowledgement. This marks the end of a session.

A randomization algorithm called Blum-Blum-Shub (BBS) is used for generating the AES key. The RSA key generation algorithm has been implemented using the Extended Euclidean algorithm. The RSA key generation needs two prime numbers, which are chosen from a list of 512 primes stored in the SOC. These key generating blocks ensure the use of a different key for every session making the system more robust, because even if a single key is obtained by a third-party, further communication sessions would still remain secure.

In order to hack the data of any session, the RSA cipher must be broken to access the AES key, followed by breaking the AES cipher to obtain the underlying data. The time required to carry this operation is significantly larger than the time for which a communication session would last, thus making the process very secure.

\section{RIVEST SHAMIR ADLEMAN}

The Rivest Shamir Adleman algorithm popularly known as RSA is a very famous public key (asymmetric) cryptographic algorithm. RSA has been used for secure exchange of the AES key. If a message ' $\mathrm{m}$ ' is to be encrypted to generate a cipher text ' $c$ ' and vice versa to get back the message using decryption then it can be obtained using the following equations:

$$
\begin{aligned}
& c \equiv m^{e} \bmod N \\
& m \equiv c^{d} \bmod N \\
& N=p * q \\
& \varphi=(p-1) *(q-1)
\end{aligned}
$$

Here, 'd' is the private key, 'e' is the public key and 'p', 'q' are any two chosen prime numbers. 


\section{A. Private Key and Key generation (Extended Euclidean Algorithm)}

The private key ' $\mathrm{d}$ ' is one of the most crucial elements in the RSA cryptosystem. If we choose two prime numbers ' $p$ ' and ' $q$ ' then we can compute ' $N$ ' and ' $\varphi$ ' using the equations [3] and [4]. After computing ' $\varphi$ ' we choose a public key 'e' using equation [5] and compute the private key ' $d$ ' using equation [6]:

$e \in\{(0, \varphi) \mid \operatorname{gcd}(e, \varphi)=1\}$

$(d * e) \bmod \varphi=1$

$d e+k \varphi=1$

For using the Extended Euclidean algorithm, we have to start by assuming that $d e+k \varphi=\mathrm{r}$. Two trivial solutions for ' $d$ ', ' $k$ ' and ' $r$ ' for this equation are $\{0,1, \varphi\}$ and $\{1,0, e\}$. Using these as $0^{\text {th }}$ and $1^{\text {st }}$ solutions we can continue finding subsequent solutions using:

$\mathrm{d}_{\mathrm{i}+1}=\mathrm{d}_{\mathrm{i}-1}-\mathrm{a}_{\mathrm{i}} \mathrm{d}_{\mathrm{i}}, \quad \mathrm{k}_{\mathrm{i}+1}=\mathrm{k}_{\mathrm{i}-1}-\mathrm{a}_{\mathrm{i}} \mathrm{k}_{\mathrm{i}}$,

$r_{i+1}=r_{i-1}-a_{i} r_{i}, \quad a_{i}=\left[r_{i-1} / r_{i}\right]$, [.] is floor function

We continue doing this till we reach a value of $i$ such that $r_{i}=1$. At this stage $d_{\mathrm{i}}$ is the modular inverse of 'e', i.e. $d=d_{i}$. If, however $d_{i}<0$, then $d=d_{i}+\varphi$.

\section{B. Implementation of Key Generation Algorithm}

The Extended Euclidean Algorithm was implemented for key generation on the Artix-7 FPGA and in python3 on Intel(R) Core(TM) i7-8550U CPU @ $1.80 \mathrm{GHz}$ machine. The simulation results have been tested and verified. The verification is very simple in case of public key and private key by equation 6 . For every new key, two prime numbers are needed. The top module of the Key Generation contains a set of 512, 64-bit prime numbers and they are selected at random using the BBS randomizer algorithm.

\section{RSA Fast Modular Exponentiation Method}

To compute $A^{B} \bmod C$ :

$A^{2} \equiv\{(A \bmod C) *(A \bmod C)\}^{*}$

$A^{4} \equiv\left\{\left(A^{2} \bmod C\right) *\left(A^{2} \bmod C\right)\right\}^{*}$

$\ldots$ and so on

For three numbers $\mathrm{A}, \mathrm{B}$ and $\mathrm{C}$ such that $\mathrm{B}$ is a power of 2 the above formula can be used. From this it can be seen that it will take $\log _{2}(\mathrm{~B})$ number of steps to get $A^{B} \bmod C$. Furthermore, if $B$ is not a power of 2 then $B$ can be written as a sum of different powers of 2 .

$B=2^{a 1}+2^{a 2}+\cdots$

Equation 12 can be obtained by representation of $B$ in the binary format. Now that the exponent $\mathrm{B}$ has been written as a sum of powers of $2, A^{B}$ can be represented as a product of numbers which are powers of $A$ such that the exponent is a power of 2 . Further 
$A^{B} \equiv A^{2^{a 1}+2^{a 2}+\cdots}$

$A^{B} \equiv A^{2^{a 1}} \bmod C * A^{2^{a 2}} \bmod C * \ldots$

*equivalence w.r.t. $\bmod (C)$.

\section{Storage Optimization}

Being a recursive algorithm, the previous values of the modular exponent are needed to calculate the subsequent values. The algorithm shows how this problem can be evaded. The code written in Verilog uses this strategy, using which only 2 variables are used over and over again, without storing the previous values. The following shows the block diagram of the algorithm:

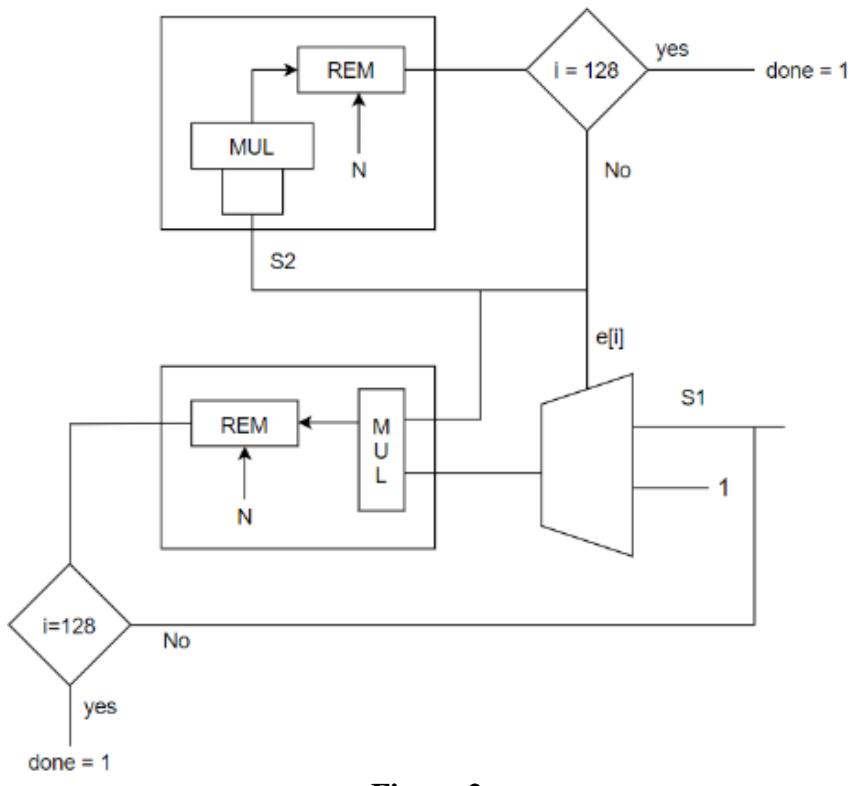

Figure 2

\section{E. The Karatsuba Multiplier}

$X=X_{1} * 2^{n / 2}+X_{r} ; Y=Y_{1} * 2^{n / 2}+Y_{r}\left(X_{1}, X_{r}\right.$ : left half bits of $X ; Y_{1}, Y_{r}$ : right half bits of $\left.Y\right)$

$\mathrm{X} * \mathrm{Y}=\left(\mathrm{X}_{1} * 2^{\mathrm{n} / 2}+\mathrm{X}_{\mathrm{r}}\right) *\left(\mathrm{Y}_{1} * 2^{\mathrm{n} / 2}+\mathrm{Y}_{\mathrm{r}}\right)($ Here $\mathrm{n} / 2$ becomes $\mathrm{n} / 4$ and so on until it becomes 32$)$

$X Y=2^{n} X_{1} Y_{1}+2^{n / 2}\left(X_{1} Y_{r}+X_{r} Y_{1}\right)+X_{r} Y_{r}$

This recursive algorithm has been implemented on an Artix-7 FPGA using the generate block in Verilog HDL. Being a recursive algorithm that halves the multiplier and multiplicand sizes each recursive step generates another hardware module. As a result the hardware blocks keep rising exponentially. In order to limit hardware utilization, the multiplier size is capped at 32. This allows for utilization of the DSP slices instead of LUTs, optimizing hardware utilization on the FPGA.

\section{F. Hardware Implementation of Fast Modular Exponentiation}


For testing, RSA-64 was carried out, i.e. the message was 64-bit long and the public and private keys were each 128-bit long generating a 128-bit cipher text. The Fast Modular Exponentiation has been implemented on Artix-7 with the help of Xilinx Vivado software in Verilog HDL, with a post-synthesis LUT Utilization of 55\% and DSP Slice Utilization of $27 \%$. With a clock period of $980 \mathrm{KHz}$, the time required for encryption and decryption for RSA-64 is $1.036 \mathrm{~ms}$ and $1.404 \mathrm{~ms}$ respectively.

\section{ADVANCED ENCRYPTION STANDARD (AES)}

The Advanced Encryption Standard also known as the Rijndael's Cipher is the standard algorithm for electronic data security. It is a symmetric key algorithm i.e. the same key is used for encryption and decryption. It allows for use of 128/192/256 bit keys. AES can be broken down into 4 main operations namely Add Round Key, Substitute Bytes, Mix Columns, and Shift Rows. The key for each round is derived from the main key using the Rijndael's key schedule as shown in Figure 3.

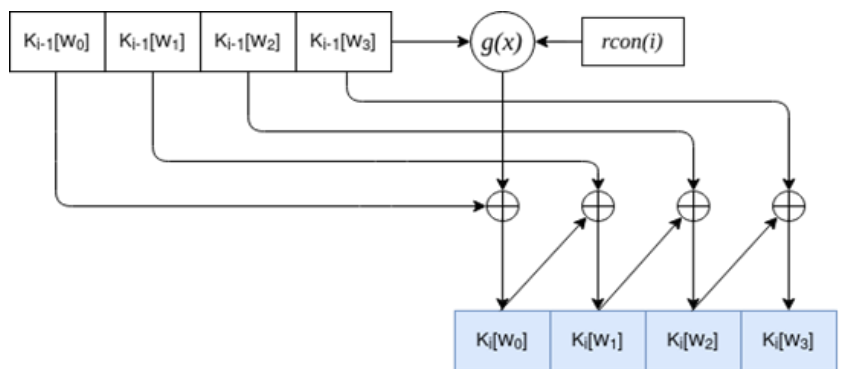

Figure 3

\section{1) Algorithm Steps}

The encryption and decryption process is broken down into 11 rounds each. A round consists of 3 to 4 operations which are performed on the data in sequential steps. Rounds 1 and 11 consists of 1 and 3 operations respectively. The operations performed on the data are as follows.

\section{A. Add Key}

The Key for Round 0 is same as the input key provided. The key for subsequent rounds are derived from the base key following the Rijndael's Algorithm. The algorithm considers the Round Constants for every round called $r \operatorname{con}(x) . r \operatorname{con}(x)$ is a 32-bit constant in a round and it is hard-coded in the hardware in the form of a lookup table. Key is represented in the format $\mathrm{K}$ $=\left[\begin{array}{lll}\mathrm{W}_{0} & \mathrm{~W}_{1} & \mathrm{~W}_{2} \\ \mathrm{~W}_{3}\end{array}\right]$ where $\mathrm{W}_{\mathrm{i}}$ is the $\mathrm{i}^{\text {th }}$ column of the Key. $g(x)$ behaves as the temp register in the following algorithm.

Key Schedule Algorithm:

if Round is $0: K(0)=K$;

for Round $i$ where is not 0 :

$$
\begin{aligned}
& \text { temp }=\left[S\left(K(i-1) W_{3}[2]\right) S\left(K(i-1) W_{3}[1]\right) S\left(K(i-1) W_{3}[0]\right) S\left(K(i-1) W_{3}[3]\right)\right] \\
& K(i) W_{0}=\text { temp } \bigoplus r \operatorname{rcon}(i) \bigoplus K(i-1) W_{0} \\
& K(i) W_{1}=\text { temp } \bigoplus r c o n(i) \bigoplus K(i) W_{0} \\
& K(i) W_{2}=\operatorname{temp} \bigoplus r \operatorname{rcon}(i) \oplus K(i) W_{1} \\
& K(i) W_{3}=\operatorname{temp} \bigoplus \operatorname{rcon}(i) \bigoplus K(i) W_{2} \\
& K e y=K(i)
\end{aligned}
$$

\section{2) Substitute Bytes}


The Substitute Bytes step consists of substituting each byte of the block with the corresponding byte from the Substitution Box. Substitution provides non-linearity to the data processing. The substitution process is a Galois Field function which is implemented by 16 lookup tables each corresponding to a byte in the block.

\section{3) Shift Rows}

The Shift Rows step consists of shuffling each row of the block by some corresponding value. This is implemented with shift registers. By following the precious steps each byte of the block can be traced to obtain a final output value. The three steps are performed over a single cycle by combining them.

\section{4) Mix Columns}

The Mix Columns consists of the Matrix Multiplication using the encryption and decryption matrix. Matrix multiplication involves multiplication with 8-bit values in Galois Field and XOR operations for addition. Further the encryption and decryption Matrix consists of values $0 \times 01$, $0 x 02,0 x 03,0 x 09, O x O B, O x O D$ and $O x O E$ only. Since the multipliers involved in the matrix multiplication are fixed, the products of any multipliers taken two at a time are stored in corresponding lookup tables. Thus the final block is a result of XOR additions which are performed over a single cycle.

\section{B. Hardware Implementation}

The hardware is implemented in the form of a micro-processing block which has its ALU, register buffer for intermediate steps and instruction set sequences for encryption and decryption respectively. The hardware design in block level is as shown in Figure 4.

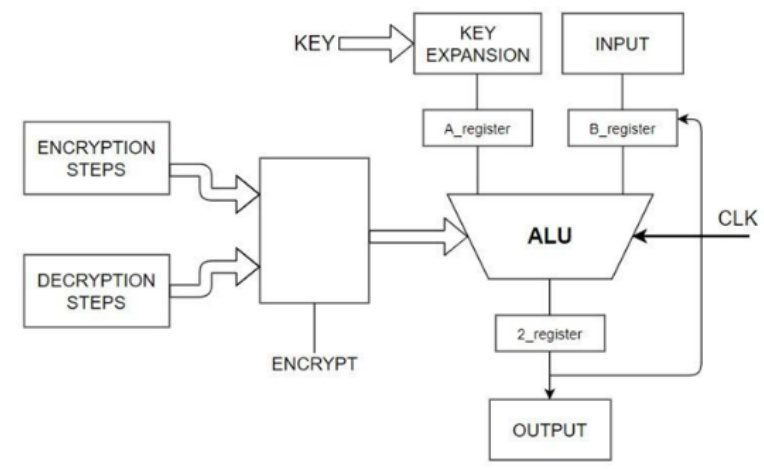

Figure 4

\section{UNIVERSAL ASYNCHRONOUS RECEIVER TRANSMITTER}

Universal Asynchronous Receiver Transmitter or UART is an electronic module for data transmission in embedded electronic systems. UART will be the terminal interface for runtime operations and program booting. UART is designed as a part of Finite State Machines for receiver and transmitter and both work independently. To support 128-bit block compatibility a higher-level state machine is used to provide 128-bit data interface. The UART is designed to work with all possible data rates till 115,200 bauds per second and can be reconfigured in runtime. 


\section{BLUM-BLUM-SHUB AlgORITHM (BBS)}

The BBS is a cryptographically secure pseudorandom number generator that generates keys that have a highly random distribution. The random keys are generated using equation 15 where ' $\mathrm{M}$ ' is a product of two 64-bit primes which are chosen from a set 512 primes stored in the SOC.

$a_{n+1}=a_{n}^{2} \bmod (M)$

\section{A. AES key generation using BBS algorithm}

With $\mathrm{a}_{0}$ as the seed, the algorithm is initiated. To ensure that the seed is truly random the system uses an independently running counter from which a seed is sampled.

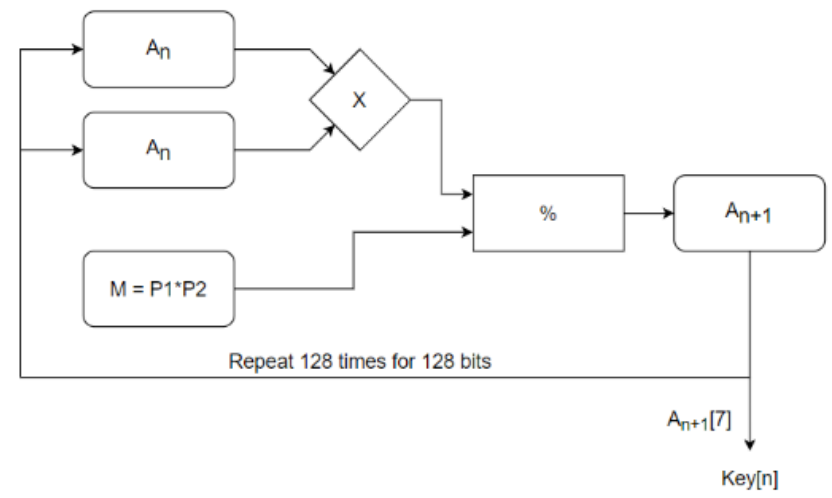

Figure 5

The algorithm works cyclically, as seen in Figure 5, to generate a random key, bit-by-bit. $a_{1}$, $\mathrm{a}_{2} \ldots \mathrm{a}_{128}$ are computed using the equation 15 and the $\mathrm{k}^{\text {th }}$ bit from each value is appended to a string to form a 128-bit number, where $\mathrm{k} \in\{0,1 . .127\}$. The final 128-bit long stream acts as the AES key. To compute the modular exponent efficiently the Montgomery algorithm has been implemented. Typically, $\mathrm{k}=0$ is chosen but using a non-zero ' $\mathrm{k}$ ' makes the generated number less deterministic.

A python code was written in order to verify the keys obtained through Verilog simulations. The keys generated were also plotted to check their randomness. We have tested several test cases, in order to determine the randomization of the algorithm, and as per the results it is evident that the keys generated are not repetitive. From figure 6, it can be seen that the distribution of keys is highly random and non-repetitive. This implementation gives a lot of freedom in terms of choosing the amount of randomization desired in addition to ease of changing the bit/bits that are chosen from each iteration, to be used in the key. Choosing a different target bit gives a completely new set of keys. 


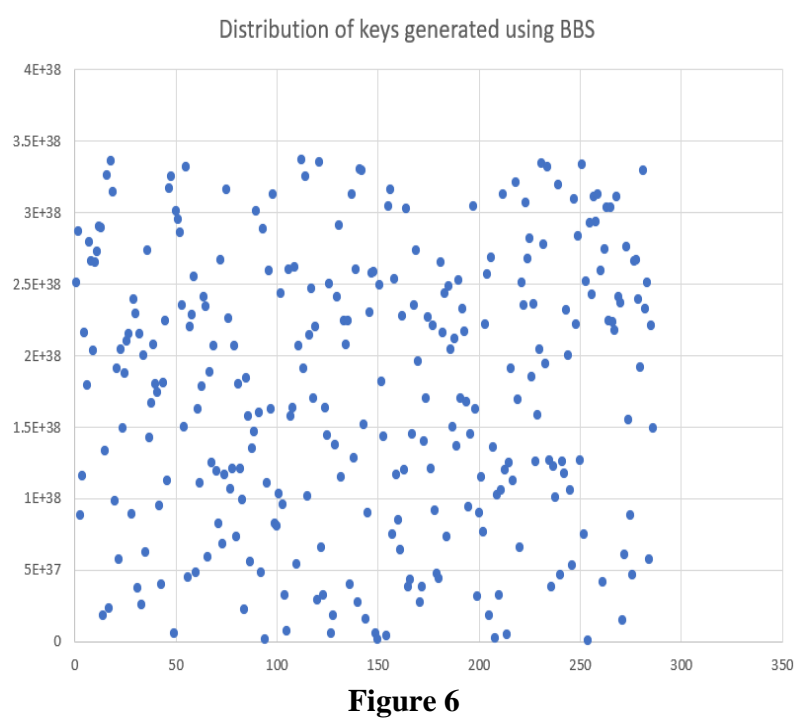

Four random sets of 8-bits are chosen from the generated random number for selection of primes, two of which are required for RSA key generation and the other two for the BBS algorithm. It can be observed that a cyclic dependence is achieved making the system selfsustaining.

\section{ARCHITECTURE}

The crypto processor is a programmable state machine with UART interface. Each instruction consists of four fields namely execution operation, operand addresses and output address each of 1 byte. It can handle a 128-bit sized data item at a time. The machine consists of clock unit, control system unit, memory units incorporating Block RAM, instruction decoder unit, key generation units and encryption/decryption units. The clock unit provides slow clock to the RSA cryptographic unit and the BBS randomizer unit. Data memory and instruction memory is separate.

\section{A. Instruction Set Architecture}

The Instruction Set architecture of our crypto processor can be outlined as follows -

1) The operands can be placed in RAM memory as well as registers.

2) Two operands are named explicitly in a typical instruction.

3) An ALU instruction operand is always placed in two registers - 'A' and ' $B$ '.

4) The operations provided in the ISA are - RSA encryption and decryption, AES encryption and decryption, RSA key generation, AES key generation, Conditional jumps, UART transmit and receive, Operand comparison, Operand increment and decrement, Sum and Difference of operands, ANDing and ORing of operands.

5) The type of each operand is 128 bit unsigned integer and its address is specified in specific bytes of the instruction.

6) It is a General Purpose Register type ISA. As our processor is a dedicated machine which provides secured communication between 2 cores, we have utilized the advantage of General Purpose Registers to store specific cryptographic parameters like cryptographic keys in specific registers for easy execution.

\section{B. Block Diagram}




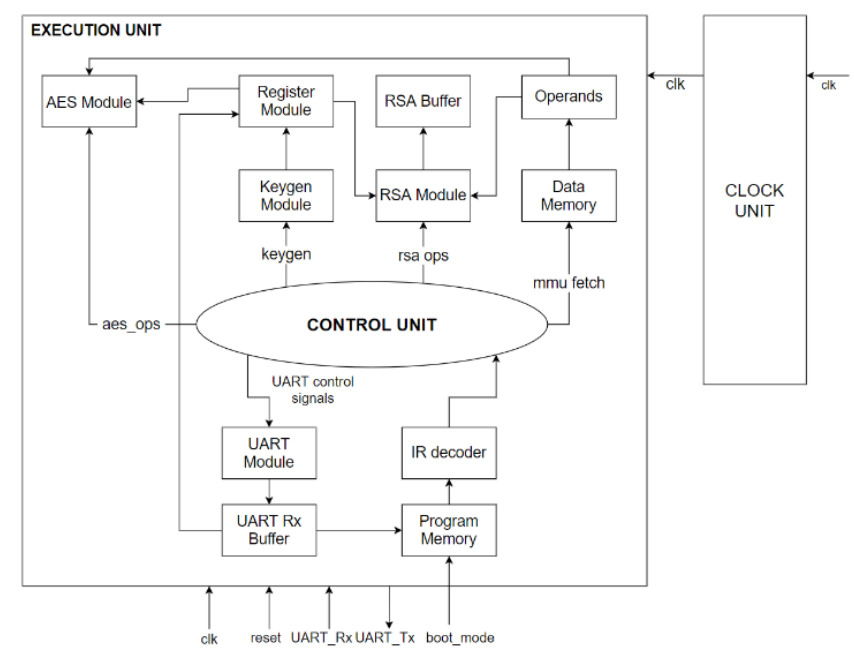

Figure 7

This figure 7 explains the system block diagram. It has inputs - clock, reset, boot-mode and also the UART transmission lines. The boot-mode input is used to differentiate between the instruction booting mode and operation execution mode. Instructions and data obtained via UART interface are placed into internal memory i.e. program memory and register module in these 2 modes respectively. The instructions are decoded and according to the specified instructions memory fetch/store operations, key generation operation, or encryption/decryption operations are performed by the respective units.

\section{Instruction flow}

The control system operates in 5 states namely, Idle, Fetch, Execute, Store and Jump. The control system has been implemented as two parallel finite state machines. One runs at positive edge of system clock and the other at the negative edge of the clock. The FSM running at positive edge is responsible for booting instructions into program memory when in boot-mode, decoding memory addresses used in store and fetch instructions and forwarding memory related control signals to the memory module. While, the FSM running at negative edge of the clock is responsible for updating the states of FSM and handling operand fetch and operation execution. 
6. RESOURCE UTILIZATION IN ARTIX-7

\begin{tabular}{|c|c|c|c|c|c|}
\hline Name & $\begin{array}{c}\text { Slice } \\
\text { LUTs } \\
(\%)\end{array}$ & $\begin{array}{c}\text { Slice } \\
\text { Registers } \\
(\%)\end{array}$ & $\begin{array}{c}\text { Block } \\
\text { RAM } \\
(\%)\end{array}$ & $\begin{array}{c}\text { Bonded } \\
\text { IOB } \\
\mathbf{( \% )}\end{array}$ & $\begin{array}{c}\text { DSP } \\
\text { (\%) }\end{array}$ \\
\hline risc & 74 & 12.46 & 1.48 & 2.30 & 27 \\
\hline eu & 74 & 12.44 & 1.48 & 0 & 27 \\
\hline alu & 10.30 & 1.70 & 0 & 0 & 0 \\
\hline aes & 5.80 & 1.50 & 0 & 0 & 0 \\
\hline ram & 0.40 & 0 & 1.48 & 0 & 0 \\
\hline rsa & 50 & 1 & 0 & 0 & 27 \\
\hline uart & 3.40 & 0.16 & 0 & 0 & 0 \\
\hline bbs & 1 & 1 & 0 & 0 & 0 \\
\hline rsa_key & 0.52 & 0.09 & 0 & 0 & 0 \\
\hline
\end{tabular}

\section{Utilization Report}

The Utilization report outlines the utilization of hardware resources from the xc7a100tcsg3242L FPGA board. The maximum utilized resource is of LUTs which are used to implement various computations in all the modules. The memory module is implemented as Block RAM. As the system is enabled with a serial UART interface, the IO buffer utilization is low. The RSA cryptographic module has been implemented such that its design can use DSP slices in place of LUTs, in order to conserve LUTs.

\section{CONCLUSIONS}

This system can be used in any application that requires secure transmission and reception. The main advantage of this system is that the system does not work with a single key or any single algorithm, rather it is a distributed system that relies on two cryptographic algorithms - RSA and AES. In the project we have developed an end to end deployment of the RSA-64 and AES128 implementation along with several randomization algorithms and fast operation algorithms.

The system developed also includes the features of a processor for handling instructions for carrying out operations related to encryption, decryption, transmission and reception. Also, a working UART module was implemented in order to enable communication with the system on chip and the main processor. The system offers flexibility since programming functionalities can be executed on the system which would be left to the user.

\section{ACKNOWLEDGEMENTS}

The authors would like to thank Dr. Vaishali Ingale for providing us with this amazing opportunity to work on this and guiding us on every step of the same. We are grateful to the Electronics and Telecommunication also for providing us with the necessary resources for completing this project.

\section{REFERENCES}


[1] A. K. Panda and K. C. Ray, "Design and FPGA Prototype of 1024- bit Blum-Blum-Shub PRBG Architecture," 2018 IEEE International Conference on Information Communication and Signal Processing (ICICSP), Singapore, 2018, pp. 38-43, doi: 10.1109/ICICSP.2018.8549715.

[2] Hassanzadeh, Alireza and Vahid Mahboubi. "A BBS Random Number Generator for Low Power Applications." International Journal of Computer Applications 131 (2015): 33-36.

[3] C. Ding, "Blum-Blum-Shub generator," in Electronics Letters, vol. 33, no. 8, pp. 677-, 10 April 1997, doi: 10.1049/el:19970440.

[4] K. Sewak, P. Rajput and A. K. Panda, "FPGA implementation of 16 bit BBS and LFSR PN sequence generator: A comparative study," 2012 IEEE Students' Conference on Electrical, Electronics and Computer Science, Bhopal, 2012, pp. 1-3, doi: 10.1109/SCEECS.2012.6184758.

[5] Rivest, Ronald L., Adi Shamir, and Leonard Adleman. "A method for obtaining digital signatures and public-key cryptosystems." Communications of the ACM 21.2 (1978): 120-126.

[6] Cao, Zhengjun, Ruizhong Wei and Xiaodong Lin. “A Fast Modular Reduction Method.” IACR Cryptol. ePrint Arch. 2014 (2014): 40.

[7] Nozaki, Hanae, et al. "Implementation of RSA algorithm based on RNS Montgomery multiplication." International Workshop on Cryptographic Hardware and Embedded Systems. Springer, Berlin, Heidelberg, 2001.

[8] Will, Mark A., and Ryan KL Ko. "Computing mod without mod." (2014).

[9] Amandeep Singh, Praveen Agarwal, Mehar Chand. "Analysis and Development of Dynamic S-box Generation" Computer Science and Information Technology 2017

[10] Tim Good and Mohammad Benaissia "AES on FPGA from fastest to smallest" Department of Electrical and Electronic Engineering, University of Sheffield

[11] Manjith Baby Chellam and Ramasubramanian Natarajan "AES Hardware Accelerator on FPGA with Improved Throughput and Resource Efficiency" Arabian Journal for Science and Engineering

[12] "Design and FPGA Implementation of UART Using Microprogrammed Controller" Mohammad Awedh, Ahmed Mueen, Scholars Journal of Engineering and Technology 